\title{
It's Ready for Targeting Muscle in Nonalcoholic Fatty Liver in This Era of Aging
}

\author{
Sae Kyung Joo and Won Kim
}

Division of Gastroenterology and Hepatology, Department of Internal Medicine, Seoul Metropolitan Government Seoul National University Boramae Medical Center, Seoul, Korea

See "Age-Related Decrease in Skeletal Muscle Mass Is an Independent Risk Factor for Incident Nonalcoholic Fatty Liver Disease: A 10-Year Retrospective Cohort Study" by Min Jung Lee, et al. on page 67, Vol. 13. No. 1, 2019

\begin{abstract}
"Sarcopenia" originates from Greek and literally means "lack of flesh." In the literature, the term is widely used as a synonym of low muscle mass; however, the international consensus guidelines for this condition (European Working Group on Sarcopenia in Older People/Foundation for the National Institutes of Health) are clear about its definition. Both groups recommend using the presence of low skeletal muscle mass and low muscle function (strength or performance) for the diagnosis of sarcopenia. $^{1}$
\end{abstract}

Sarcopenia is a more complex condition than can be described by either single factor. ${ }^{1}$ There are multiple factors that may contribute to sarcopenia in patients with nonalcoholic fatty liver disease (NAFLD). The causes that are responsible for sarcopenia in NAFLD patients include increased insulin resistance, changes in levels of myokines such as growth differentiation factor 15 (GDF-15), ${ }^{2}$ elevated chronic inflammation markers, and lower vitamin D levels.

When assessing sarcopenia, muscle mass is usually normalized to weight $(\mathrm{kg})$ or height squared $\left(\mathrm{m}^{2}\right)$. With dual-energy Xray absorptiometry (DEXA), which is one of the recognized gold standards in clinical assessment of sarcopenia (the other being computed tomography/magnetic resonance imaging), the most recent muscle mass index, validated in multiethnic populations, is determined as follows: appendicular skeletal muscle mass (kg) divided by body mass index $\left(\mathrm{kg} / \mathrm{m}^{2}\right)^{3}$

Several study groups have investigated an association between muscle mass or sarcopenia and NAFLD in cross-sectional settings. ${ }^{4-6}$ Moreover, it has recently been reported that reduced skeletal muscle mass increases the risk of the onset of NAFLD ${ }^{4,6,7}$ as well as the risk of liver fibrosis. ${ }^{7}$ Shida et al. ${ }^{8}$ have explored whether a reduction in skeletal muscle mass and an increase in visceral fat mass are associated with the risk of the onset of NAFLD and serve as important risk factors involved in the progression of liver fibrosis. Recently, Kim et al. ${ }^{9}$ have observed that an increase in relative skeletal muscle mass over time may be beneficial for preventing NAFLD development or resolving NAFLD in a dose-dependent manner, independent of the baseline skeletal muscle index.

In the current issue, Lee et al. ${ }^{10}$ have found that a progressive increase in the fat mass and a loss of skeletal muscle mass with aging were significantly associated with incident NAFLD in the general population. The authors assessed aging-related changes in the body composition over a 10-year period in a relatively large population. In particular, they showed that a decreased muscle mass was significantly associated with incident NAFLD in nonobese but not in obese subjects after adjustment for various metabolic risk factors.

The skeletal muscle mass was measured by means of bioelectrical impedance analysis, although it was not as precise as direct measures of muscle mass. In subgroup analyses, the absolute change in appendicular skeletal muscle mass was significantly associated with incident NAFLD, particularly in the nonobese and female subgroups. These results are notable because nonobese NAFLD is more frequently found in Asian countries than in Western regions.

Meanwhile, important nutritional and body compositional variables, confounding or mediating the associations among obesity, sarcopenia, and NAFLD, such as the vitamin D level and visceral adiposity, were not analyzed in the current study. In addition, the data should have been adjusted for the physical

Correspondence to: Won Kim

Division of Gastroenterology and Hepatology, Department of Internal Medicine, Seoul Metropolitan Government Seoul National University Boramae Medical Center, Seoul National University College of Medicine, 20 Boramae-ro 5-gil, Dongjak-gu, Seoul 07061, Korea

Tel: +82-2-870-2233, Fax: +82-2-831-2826, E-mail: drwon1@snu.ac.kr pISSN 1976-2283 eISSN 2005-1212 https://doi.org/10.5009/gnl18483

@ This is an Open Access article distributed under the terms of the Creative Commons Attribution Non-Commercial License (http://creativecommons.org/licenses/by-nc/4.0) which permits unrestricted non-commercial use, distribution, and reproduction in any medium, provided the original work is properly cited. 
activity level, which is related to the axial muscle mass, since unlike the core muscle mass, the axial muscle mass is affected by physical activity. Moreover, the study only presented odds ratios but not hazard ratios for incident NAFLD, without any data on regressed NAFLD, although the researchers followed the study population for 10 years. Therefore, it was difficult to accurately estimate the incidence of NAFLD or predict the regression of NAFLD during the study period.

Despite these limitations, it was a well-designed, retrospective, single-institution study including a large community-based population. It unveiled the association of the absolute change in skeletal muscle mass with incident NAFLD by using longitudinal cohort data. Additionally, this study showed a stronger association between decreasing skeletal muscle mass and incident NAFLD in nonobese subjects, highlighting that sarcopenia as well as visceral adiposity, dietary preferences (e.g., a highfructose diet), and genetic polymorphisms are well-known metabolic or genetic risk factors for nonobese or lean NAFLD. However, further studies involving detailed histological assessment and adjunct evaluation of muscle quality are warranted to clarify the causal relationship between sarcopenia or sarcopenic obesity and NAFLD development or progression.

\section{CONFLICTS OF INTEREST}

No potential conflict of interest relevant to this article was reported.

\section{ACKNOWLEDGEMENTS}

This work was supported by a National Research Foundation of Korea (NRF) grant funded by the Korea government (Ministry of Education, Science and Technology; MEST) (2016R1D1A1B04934590) and the Korea Health Technology R\&D Project through the Korea Health Industry Development Institute (KHIDI) funded by the Ministry of Health \& Welfare, Republic of Korea (HI17C0912).

\section{REFERENCES}

1. Cruz-Jentoft AJ, Baeyens JP, Bauer JM, et al. Sarcopenia: European consensus on definition and diagnosis: report of the European Working Group on Sarcopenia in Older People. Age Ageing 2010;39:412-423.

2. Koo BK, Um SH, Seo DS, et al. Growth differentiation factor 15 predicts advanced fibrosis in biopsy-proven non-alcoholic fatty liver disease. Liver Int 2018;38:695-705.

3. Studenski SA, Peters KW, Alley DE, et al. The FNIH sarcopenia project: rationale, study description, conference recommendations, and final estimates. J Gerontol A Biol Sci Med Sci 2014;69:547558

4. Hong HC, Hwang SY, Choi HY, et al. Relationship between sarcopenia and nonalcoholic fatty liver disease: the Korean Sarcopenic Obesity Study. Hepatology 2014;59:1772-1778.

5. Koo BK, Kim D, Joo SK, et al. Sarcopenia is an independent risk factor for non-alcoholic steatohepatitis and significant fibrosis. J Hepatol 2017;66:123-131.

6. Lee YH, Jung KS, Kim SU, et al. Sarcopaenia is associated with NAFLD independently of obesity and insulin resistance: nationwide surveys (KNHANES 2008-2011). J Hepatol 2015;63:486-493.

7. Lee YH, Kim SU, Song K, et al. Sarcopenia is associated with significant liver fibrosis independently of obesity and insulin resistance in nonalcoholic fatty liver disease: nationwide surveys (KNHANES 2008-2011). Hepatology 2016;63:776-786.

8. Shida T, Akiyama K, Oh S, et al. Skeletal muscle mass to visceral fat area ratio is an important determinant affecting hepatic conditions of non-alcoholic fatty liver disease. J Gastroenterol 2018;53:535-547.

9. Kim G, Lee SE, Lee YB, et al. Relationship between relative skeletal muscle mass and nonalcoholic fatty liver disease: a 7-year longitudinal study. Hepatology 2018;68:1755-1768.

10. Lee MJ, Kim EH, Bae SJ, et al. Age-related decrease in skeletal muscle mass is an independent risk factor for incident nonalcoholic fatty liver disease: a 10-year retrospective cohort study. Gut Liver 2019;13:67-76. 